\title{
Supraphrenic herniation of perinephric fat
}

\author{
B. T. LE ROUX
}

From the Thoracic Surgical Unit, The Royal Infirmary, Edinburgh

The diagnosis attached pre-operatively to wellcircumscribed peripheral intrathoracic opacities is often no more than an educated guess, and before an accurate histological diagnosis can be made it is usually necessary to submit to thoracotomy the patient who harbours the lesion responsible for such a shadow. The gamut of radiographic and surgical investigations so rarely provides an answer complete enough to obviate diagnostic thoracotomy that these are often omitted. In relatively rare circumstances, however, investigation may establish beyond any reasonable doubt a diagnosis which makes thoracotomy-either diagnostic or therapeutic-unnecessary. The supraphrenic herniation of perinephric fat is an example of a lesion, the diagnosis of which can be unequivocally established without thoracotomy.

Three patients with this lesion have been managed in the Thoracic Surgical Unit in Edinburgh in the last 15 years. All were symptomless, obese men in the seventh decade, and in all the shadow had been found at routine radiography for an unrelated reason. The first patient, whose chest radiographs are shown as Fig. 1, presented in 1953 and was submitted to diagnostic thoracotomy after preliminary investigation which included fluoroscopy, tomography, and bronchoscopy, and with a pre-operative diagnosis no more precise than intrathoracic, possibly pleural, tumour. At left thoracotomy a mushroom-shaped mass of perinephric fat was found to have herniated extrapleurally into the left hemithorax through a circular defect in the postero-lateral, muscular part of the diaphragm. The defect was $2 \mathrm{~cm}$. in diameter and lay $3 \mathrm{~cm}$. from the peripheral attachment of the diaphragm. The fat was amputated and the defect closed; recovery was uneventful, and the patient remains well.

The second patient (Fig. 2) presented in 1962. In him the provisional diagnosis of intrathoracic herniation of perinephric fat was made because of the similarity between the radiographic appearances in this and the first case. As with the first patient, the opacity could not be separated from the diaphragm at fluoroscopy. When a pneumo- peritoneum was induced air did not enter the opacity, and the left dome of the diaphragm was $\tilde{O}$ clearly outlined (Fig. 3 (above)). A pneumothorax was induced (Fig. 3 (below)), and the opacity was shown to be separate from the lung. At thoracoscopy the tumour responsible for the shadow was 을 seen, from its colour and its behaviour when $\rightarrow$ displaced with a probe, to be fat; the fat could be so displaced as to identify the defect in the diaphragm. Further surgical action was not taken, and the patient remains well with the opacity unchanged.

The third patient presented in 1964. The postero-anterior and lateral chest radiographs (Fig. 4) were almost indistinguishable from those of the second patient. After the pre-sacral injec- $\mathbb{D}$ tion of air the opacity was shown to contain translucencies, and the kidney shadow was clearly seen to lie below the diaphragm. A pneumothorax was induced, and the subsequent radiographic appearances and those at thoracoscopy were similar to those seen in the second patient.

The radiographic appearances in these three patients were similar; in the second and third they were almost indistinguishable. None of the opacities was dense, and the relative lack of density suggested that they might be the shadows of fat, an observation validly made elsewhere in the chest, as, for example, in the anterior cardiophrenic angle, where the herniation of extraperitoneal fat through the right foramen of Morgagni can simulate precisely a pericardial of coelomic cyst, except for the relative lack of $N$ density of the shadow of fat (le Roux, 1960). In N all three patients the opacity was inseparable from $\omega$ the diaphragm at fluoroscopy. A technically poor film may suggest that a peripheral pulmonary

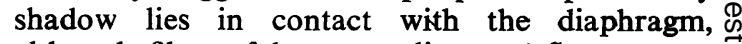
although films of better quality, and fluoroscopy, later demonstrate that the lesion is within the lung (Fig. 5). The hazards of pre-sacral insuffla- $\overrightarrow{\mathbb{D}}$ tion of air are said to be negligible when the $\frac{?}{\mathbb{D}}$ investigation is undertaken by an expert. The $\stackrel{2}{2}$ induction of a pneumothorax carries some danger. but less than that of thoracotomy in the 


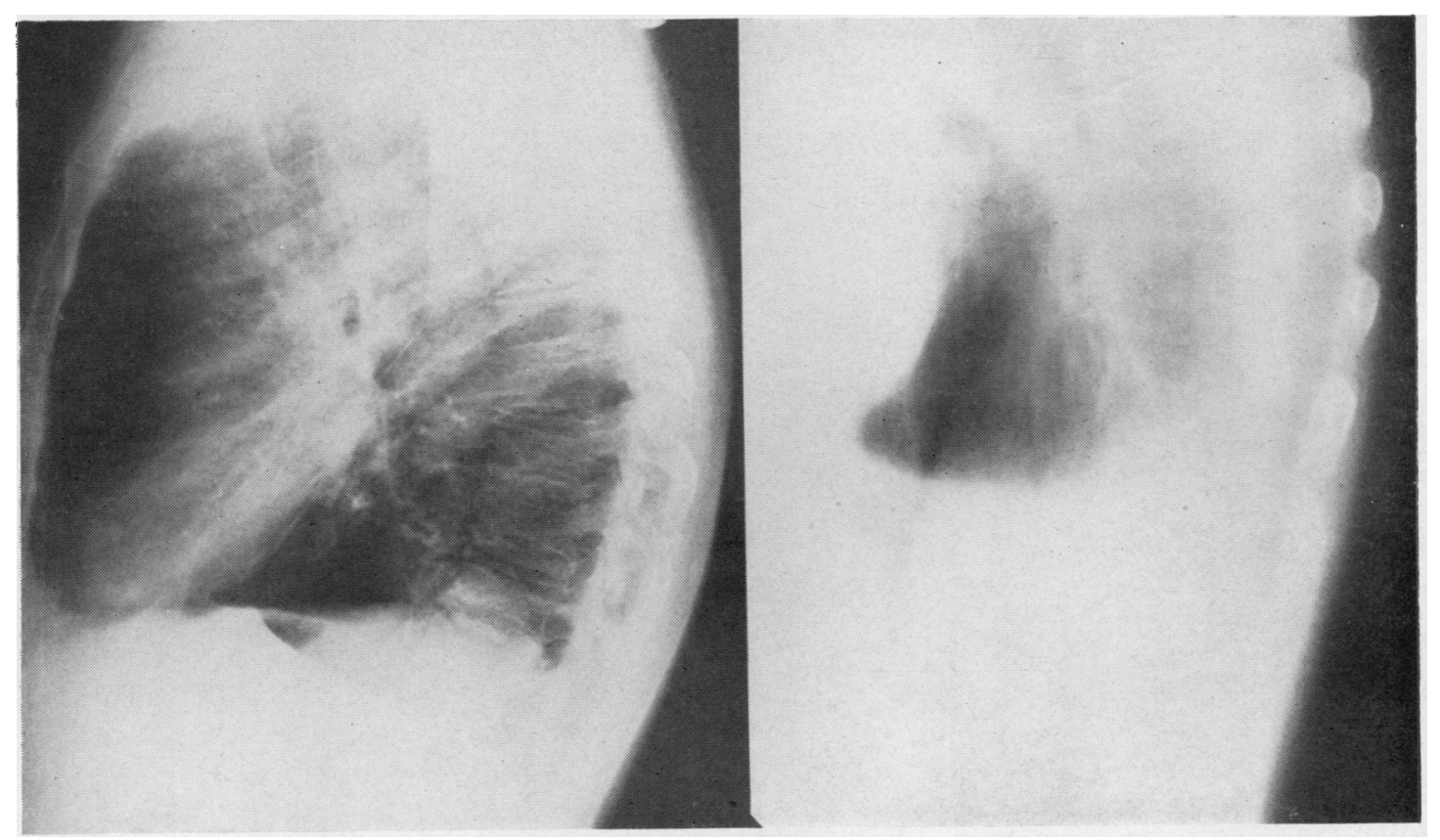

FIG. 1. Case 1. Left lateral radiograph and lateral tomogram.

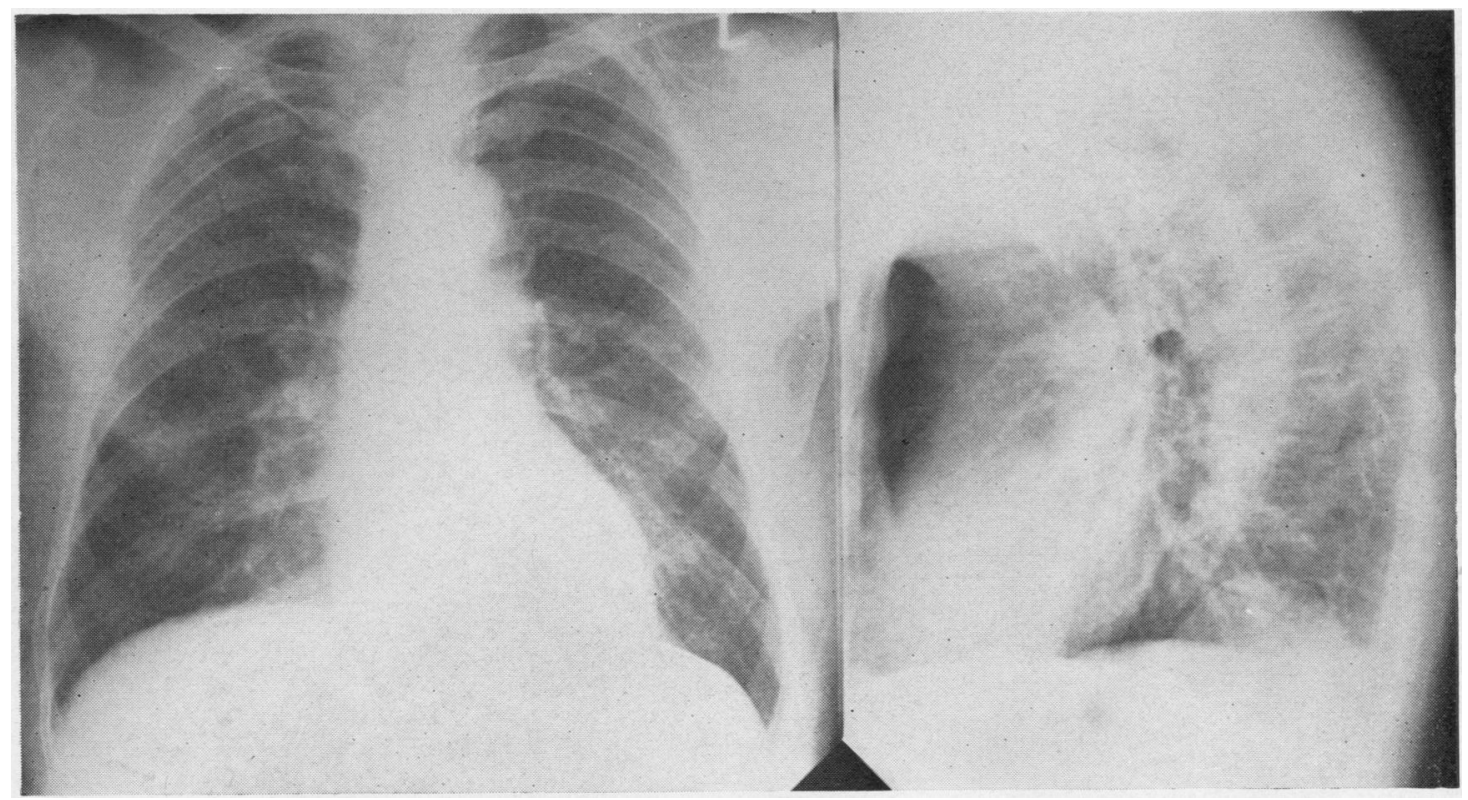

1IG. 2. Case 2. Postero-anterior and left lateral radiographs. 
FIG. 3. Case 2. (Above) A pneumoperitoneum has been induced, and air does not enter the left posterior supraphrenic shadow;(below) a pneumothorax has been induced, and the opacity is separate from the lung. 


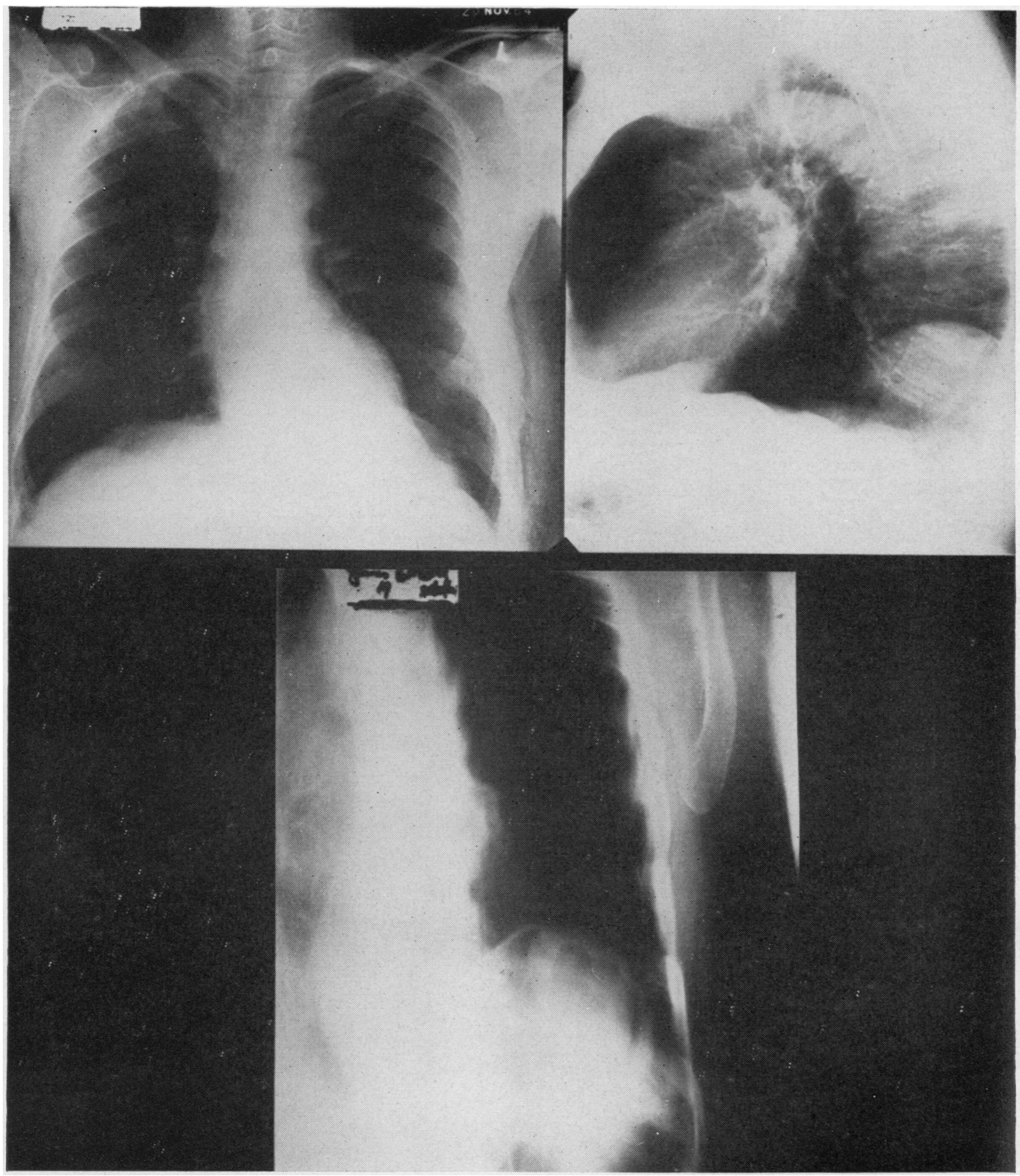

FIG. 4. Case 3. (Above) The opacity, which lies posteriorly in the left costophrenic angle, is seen to differ little from that shown in Fig. 2; (below) shows translucencies within the shadow after the pre-sacral in sufflation of air. 


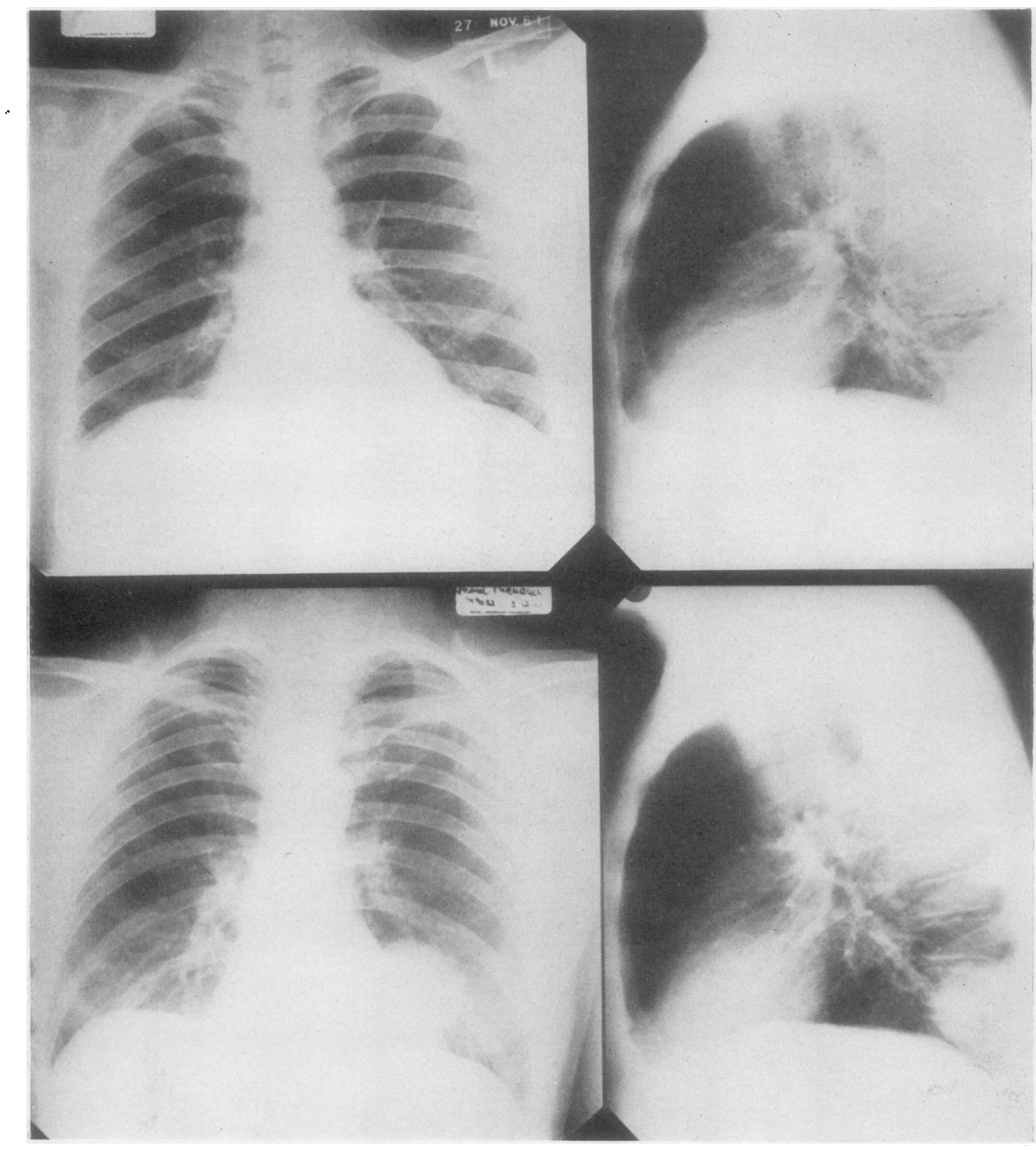

FIG. 5. The upper and lower pairs of radiographs were made for the same patient on the same day. The upper pair is technically poor and was not made at the end of inspiration. Technically better films made in full inspiration (below) show clearly that the opacity is pulmonary. 
elderly and obese. Diagnostic thoracoscopy in expert hands is not known to be associated with either morbidity or mortality.

If another patient who is elderly and obese should present to this unit with an opacity similar to those illustrated-an opacity situated in the left posterior costophrenic angle, of a density acceptable as that of fat, and with an outline of such sharpness as to suggest a pleural covering -after making postero-anterior and lateral films we should limit investigations to fluoroscopy and, if the opacity were inseparable from the diaphragm, the pre-sacral insufflation of air. If after this last investigation the opacity was seen to contain translucencies, further investigation would not be undertaken.

The defect in the diaphragm through which herniation of perinephric fat is permitted is in the same situation and looks the same as that found on the right in the reported cases of hetero- topic intrathoracic liver (le Roux, 1961) and probably represents persistence of part of the pleuro-peritoneal canal.

\section{SUMMARY}

Herniation of perinephric fat through the left dome of the diaphragm casts a radiographic shadow readily recognizable on plain films of the chest, and the diagnosis can be established at fluoroscopy and by the pre-sacral insufflation of air. If further confirmation of the diagnosis is required, the fatty hernia is readily recognizable at thoracoscopy. Diagnostic thoracotomy can therefore be avoided in a patient who presents with this abnormality.

\section{REFERENCES}

le Roux, B. T. (1960). Spherical radiographic opacities in the anterior cardiophrenic angle. J. roy. Coll. Surg. Edinb., 5, 158. (1961). Heterotopic intra-thoracic liver. Thorax, 16, 68. 